\title{
Generation and Transcriptome Profiling of S1r1-d7 and S1r1-d8 Mutant Lines with a New Semi-Dominant Dwarf Allele of SLR1 Using the CRISPR/Cas9 System in Rice
}

\author{
Yu Jin Jung ${ }^{1,2}$, Jong Hee Kim ${ }^{1}$, Hyo Ju Lee ${ }^{1}$, Dong Hyun Kim ${ }^{1}$, Jihyeon Yu ${ }^{3}$, Sangsu Bae ${ }^{3}$, \\ Yong-Gu Cho ${ }^{4}$ and Kwon Kyoo Kang ${ }^{1,2, *}$ \\ 1 Division of Horticultural Biotechnology, Hankyong National University, Anseong 17579, Korea; \\ yuyu1216@hknu.ac.kr (Y.J.J.); gllmon@naver.com (J.H.K.); ju950114@naver.com (H.J.L.); \\ skullmask@naver.com (D.H.K.) \\ 2 Institute of Genetic Engineering, Hankyong National University, Anseong 17579, Korea \\ 3 Department of Chemistry, Hanyang University, Seoul 04763, Korea; muner8146@gmail.com (J.Y.); \\ sangsubae@hanyang.ac.kr (S.B.) \\ 4 Department of Crop Science, Chungbuk National University, Cheongju 28644, Korea; ygcho@chungbuk.ac.kr \\ * Correspondence: kykang@hknu.ac.kr; Tel.: +82-31-670-5104
}

Received: 30 June 2020; Accepted: 29 July 2020; Published: 31 July 2020

\begin{abstract}
The rice SLR1 gene encodes the DELLA protein (protein with DELLA amino acid motif), and a loss-of-function mutation is dwarfed by inhibiting plant growth. We generate slr1-d mutants with a semi-dominant dwarf phenotype to target mutations of the DELLA/TVHYNP domain using CRISPR/Cas9 genome editing in rice. Sixteen genetic edited lines out of 31 transgenic plants were generated. Deep sequencing results showed that the mutants had six different mutation types at the target site of the TVHYNP domain of the SLR1 gene. The homo-edited plants selected individuals without DNA (T-DNA) transcribed by segregation in the T1 generation. The slr1-d7 and slr1-d8 plants caused a gibberellin (GA)-insensitive dwarf phenotype with shrunken leaves and shortened internodes. A genome-wide gene expression analysis by RNA-seq indicated that the expression levels of two GA-related genes, $\mathrm{GA}_{20} \mathrm{OX}_{2}$ (Gibberellin oxidase) and $\mathrm{GA}_{3} \mathrm{OX}_{2}$, were increased in the edited mutant plants, suggesting that $\mathrm{GA}_{20} \mathrm{OX}_{2}$ acts as a convert of $\mathrm{GA}_{12}$ signaling. These mutant plants are required by altering GA responses, at least partially by a defect in the phytohormone signaling system process and prevented cell elongation. The new mutants, namely, the slr1-d7 and slr1-d8 lines, are valuable semi-dominant dwarf alleles with potential application value for molecule breeding using the CRISPR/Cas9 system in rice.
\end{abstract}

Keywords: CRISPR/Cas9; GA; DELLA/TVHYNP; Dwarf; $G A_{20} O X_{2}$; GA signaling

\section{Introduction}

Rice, being one of the major food crops consumed by nearly half of the world's population, is grown annually at about 4.5 million hectares. Rice consumption per capita is particularly high in Asia, where it provides 60-70\% calories per day (Food and Agriculture Organization (FAO), 2004). Therefore, among the breeding program priorities of rice breeders is to improve its tolerance to abiotic stress, such as tolerance to lodging. To date, the properties of many dwarf mutants found in plants have been associated with genes on the biosynthesis and signaling pathways of gibberellin (GA), indole-3-acetic acid (IAA), brassinolide (BR), and other hormones [1]. GA is one of the important plant hormones acting as a group of diterpenoid compounds that regulate during various growth 
and development processes in the higher plants, including stem elongation, germination, dormancy, flowering, flower development, leaf, and fruit aging [1-3]. The phenotypes of mutants deficient in GA biosynthesis or signaling usually exhibit dark green and rough leaves in rice [4]. So far, several genes related to defective mutants on the GA biosynthetic pathway, namely, $d 18, d 35$, sd1, and eui, have been isolated and characterized in rice [5-8]. Molecular genetic studies of GA-sensitive rice and Arabidopsis mutants have identified important factors for GA signaling, which seems to be well conserved among flowering plants $[9,10]$. The most important regulator of the GA signaling pathway is the DELLA protein, which is known as the repressor of GA action [11,12]. The DELLA proteins belong to the GRAS family as a transcription factor and are known to contain the N-terminal DELLA/TVHYNP amino acid motif and the C-terminal GRAS domain $[13,14]$. In addition, the genes encoding the GA receptor GA-INSENSITIVE DWARF1 (GID1), the F-box protein GA-INSENSITIVE DWARF2 (GID2) and the DELLA protein have been cloned, and an integrated GA signal transduction pathway has emerged [15-17]. Furthermore, it has been reported that DELLA family proteins interact with growth-related transcription factors such as PIF (phytochrome interaction factor) to control plant cell and organ size [18]. In general, GID1-GA-DELLA complexes in plant cells recognize GA by receptors. However, in the case of rice, the F-box protein of GID2 additionally interacts with the DELLA protein, which is polyubiquitinated by E3 ubiquitin-ligase (GID2) and then degraded through the $26 \mathrm{~S}$ proteasome [19]. It is known that internode elongation is facilitated by GA signaling through GID1 and the DELLA protein in rice [20]. To date, accumulating evidence highlighted the N-terminus of DELLA as necessary for the inhibition of GA action. It has also recently been shown that DELLA $\mathrm{N}$-terminus is required to interact with the GA receptor GID1 and consequent degradation [21]. In rice, the slr1-d1, -d2, -d 3, -d4, -d5 and-d6 mutants in the GA signal transduction inhibitor DELLA protein $\mathrm{N}$-terminal region consequently result in a dominant, semi-dwarf phenotype [22]. These mutants are known to have an amino acid modified by one bp substitution in the conserved DELLA/TVHYNP domain of the DELLA protein [22].

The CRISPR/Cas9 system, a recently developed genome modification tool, has been widely used for genome editing of several major crops due to its high accuracy and efficiency [23,24]. Furthermore, CRISPR/Cas9 has not only been used to knock out target genes in cells but also to introduce fragments of a certain size into the gene $[25,26]$.

In this study, the CRISPR/Cas9 system was employed for targeting the TVHYNP domain of the OsSLR1 gene, known as the DELLA protein. A total of six homozygous edited plants with new different allelic variants, namely, slr1-d7, slr1-d8, slr1-d9, slr1-d10, slr1-d11, and slr1-d12, showed dwarfism. In addition, mutants slr1-d7 and slr1-d8 were further investigated at transcriptome levels using RNA-sequencing.

\section{Results}

\subsection{Editing of the TVHYNP Domain Encoding the OsSLR1 Gene and the CRISPR/Cas9 System}

According to the structure of the OsSLR1 gene, DELLA and TVHYNP domains are well conserved at the N-terminus (Figure 1A, Supplementary Figure S1 and S2). Sixteen mutants were identified by single guide RNA (sgRNA) region which targeted the OsSLR1 gene in the positive transgenic $\mathrm{T}_{0}$ plants (Supplementary Table S1, Figure S3 and S4). Deep sequence analyses detected 6 homozygous mutations, 2 heterozygous mutations, and 8 bi-allelic mutations (Supplementary Table S2). All the $\mathrm{T}_{0}$ mutants were dwarf, producing many tillers. Six homozygous mutants were identified, among which four were characterized by few bp deletions and two by a few bp insertions. Specifically, the following were observed: a 3-bp deletion and mutant named slr1-d7, a 1-bp deletion that was designated as slr1-d8, a 5-bp deletion named slr1d9 and a 14-bp deletion called slr1-d10. The insertion mutants were a T-insertion named slr1d11 and a C-insertion named slr1-d12 (Figure 1B). Theoretically, the slr-d7 mutant encodes a protein without serine (Ser, S) and the slr1-d8 slr1-d12 are knockout mutants with a stop codon that cannot encode the protein (Figure 1C). A single-base deletion and insertion are 
predicted to cause a frameshift, resulting in the knockout of the OsSLR1 gene. However, all the mutations did not affect the core sequence TVHYNP domain of the OsSLR1 gene. The sgRNA was also investigated using Cas-OFFinder (http://www.rgenome.net/cas-offinder/) [27], and two potential off-target sites were chosen. Interestingly, no mutations were detected in these loci. These mutations are either untranslated or modified SLR1 proteins and have different mutant sites compared to the previously reported slr1-d1 slr1-d6 allele [28-30].

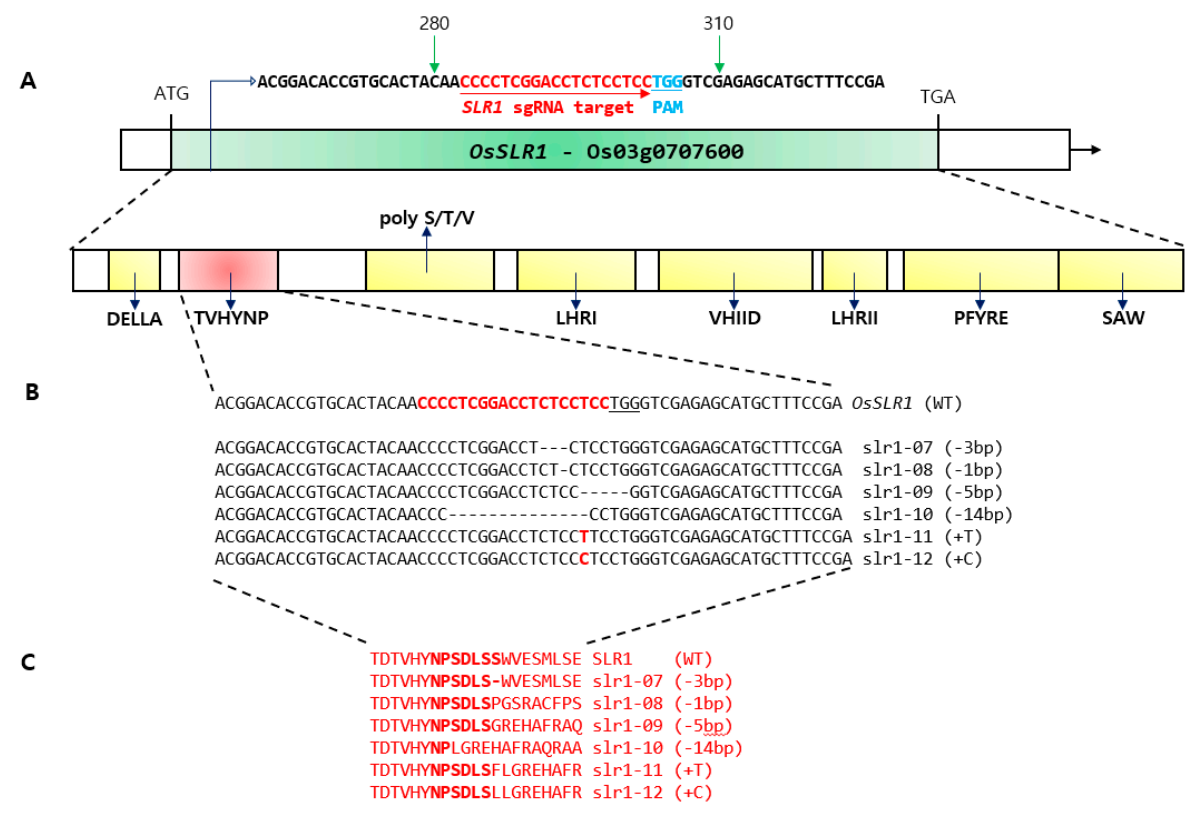

Figure 1. Genome editing in the rice OsSLR1 gene. (A) Design of single guide RNA (sgRNA) sites in the TVHYNP motif; the nuclease cleavage site is represented by the red arrow and the Protospacer Adjacent Motif (PAM) (NGG) appears in blue. DELLA protein organization representing the conserved domains. (B) Nucleotide sequence alignment by deep sequence analysis of the sgRNA target region in six mutant lines of transformed rice plants. Deletion and insertion indicated by dash and red letters, respectively. (C) Amino acid sequences of the target region in six mutant lines.

\subsection{New Allelic Slr-d7 Slr-d8 Mutant Plants Showed Dwarfing}

The new dwarf mutants (slr1-d7 slr1-d12) showed several deficiencies in addition to reducing plant size (Supplementary Figure S5). Compared to the wild type (WT), these mutants had a slow growth rate, showed dwarfed and shriveled leaves (Figure 2A). The stomata are the key channels that regulate gas exchange and water evaporation in the leaves. As a result of observing stomata sizes by SEM (scanning electron microscopy) images, the slr1-d7 and slr1-d8 lines were smaller than that of WT (Figure 2B). To observe cytological differences in the stem internodes of these mutants, paraffin sections of the stem internodes were investigated from two mutants (slr1-d7 and slr1-d8) and WT. These dwarf mutants showed that the cell size was significantly reduced, and the internode thickened as the cell layer was increased (Figure 2C). In addition, the length of all internodes of the slr1-d7 and slr1-d8 lines were reduced compared to WT (Figure 2D). These results are similar to the characteristics of $d n$-type rice dwarf mutants previously reported by Takeda [31]. Thus, slr1-d7 and slr1-d8 lines were semi-dominant dwarf mutants, indicating that a decrease in cell length may be a direct cause of shortened culm length in dwarf mutant plants. Furthermore, to know the cause of dwarfism, the length of the leaf sheath was measured according to $\mathrm{GA}_{3}$ concentration treatment in slr-d7, slr-d8, and WT. The results showed that the slr- $\mathrm{d} 7$ and slr-d8 variants produced a more extended leaf sheath following $\mathrm{GA}_{3}$ treatment, but a reduced length extension compared to WT (Figure 2E). 
^

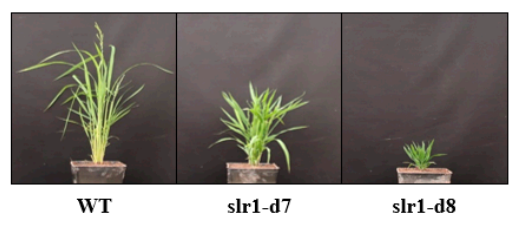

B
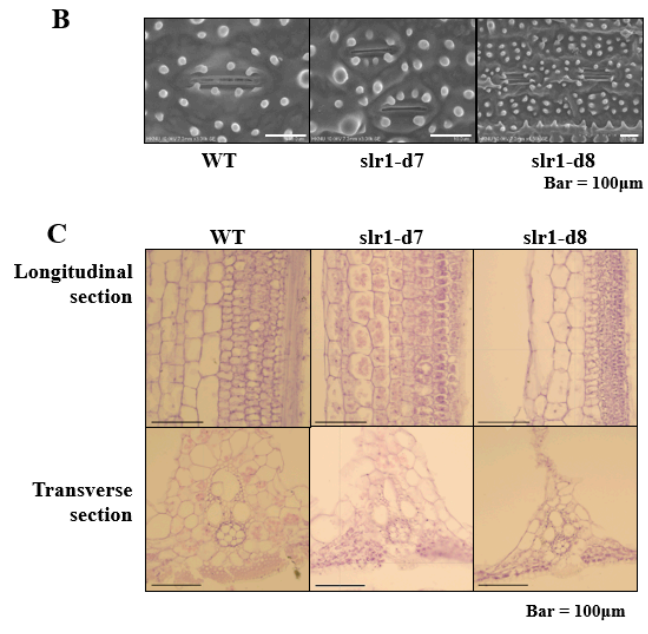

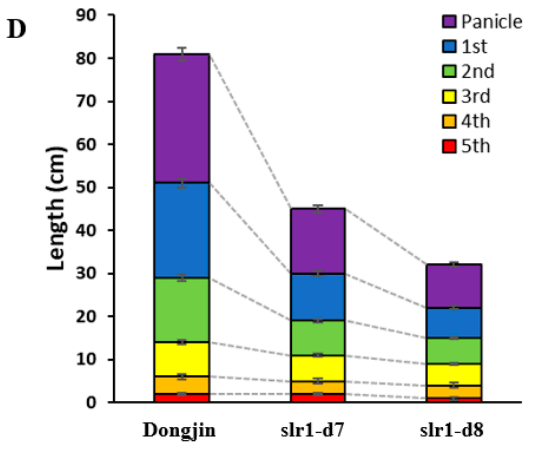

$\mathbf{E}$

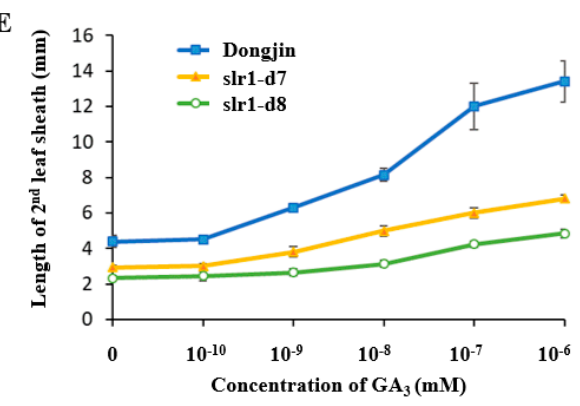

Figure 2. Phenotypic analysis of wild-type (WT) and slr1 mutant plants. (A) Phenotype of mature WT and mutant plant lines. (B) SEM (scanning electron microscopy) images of rice stomata in slr1-d7, slr1-d8 and WT. (C) Longitudinal tissue sections of the main stem at the mature stage in WT and slr1 mutant using paraffin section. Bar: $100 \mu \mathrm{m}$. (D) Length of internodes in slr1-d7, slr1-d8 and WT. (E) Elongation of the second leaf sheath of slr1-d7 and slr1-d8 in response to exogenous treatment with different concentrations of $\mathrm{GA}_{3}$. Error bars are SD from the mean $(n=3)$.

\subsection{Altered Transcriptome Profiling in Slr-d7 and Slr-8 Mutants}

To understand the impact of dwarfism on gene expression at the whole-genome level, RNA-Seq was conducted to detect transcription profiling changes in WT, slr1-d7 and slr1-d8 lines. RNA-seq results showed that gene expression was altered significantly between WT and the slr1-d7 and slr1-d8 lines (Figure 3A). There are 214 genes upregulated and 154 genes downregulated in the slr1-d7 mutant compared with WT plants. By comparison, 334 genes were upregulated and 104 genes were downregulated in the slr1-d8 line (Figure 3B). Venn diagram analysis revealed 806 genes expressed in both WT and slr1-d7 or slr1-d8 mutants, which may explain the effects of knocking out SLR1 on plants (Figure 3B,C). Gene ontology (GO) enrichment analysis of the 806 annotated up- and down-regulated genes identified 193 significantly (false discovery rate (FDR < 0.05)) enriched GO terms for the biological process, cellular component, and molecular function categories (Figure 4). Within the biological process category, the enriched differentially expressed genes (DEGs) were mainly associated with the response to the oligopeptide transport (GO:0006857), the intracellular protein transport (GO:0006886), karrikin (GO:0080167), and salt stress (GO:0009651). Within the cellular component category, the enriched DEGs were mainly associated with the plasma membrane (GO:0005886), the membrane (GO:0016020), cytosol (GO:0005829), and the integral component of the membrane (GO:0016021). Within the molecular function category, the DEGs were associated with protein serine/threonine kinase activity (GO:0004674), ATP binding (GO:0005524), and protein binding (GO:0005515) (Supplementary Table S3). To confirm the results from the RNA-seq analysis, 38 DEGs in the enriched GO terms were selected in the slr1-d7 and slr1-d8 lines, and their expression levels were confirmed by qRT-PCR analysis. The qRT-PCR results showed that the transcription levels of these genes were consistent with the RNA-seq results (Figure 5). These results indicated that dwarfism of the slr1-d7 and slr1-d8 lines mediates gene expression levels involved in regulating the plant hormone (GA, salicylic acid (SA), jasmonate (JA), IAA, cytokinin (CT) and ethylene (ET)) metabolism, signal transduction and transport (Supplementary Table S4). 
A

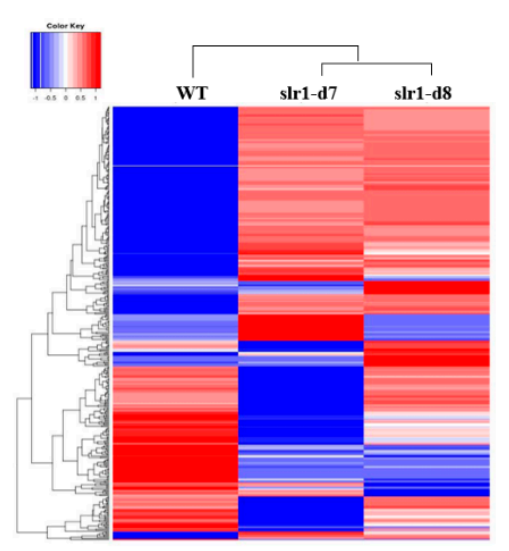

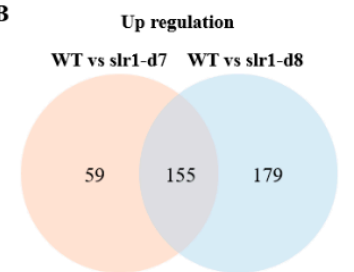

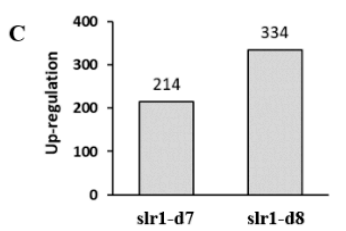

Down regulation
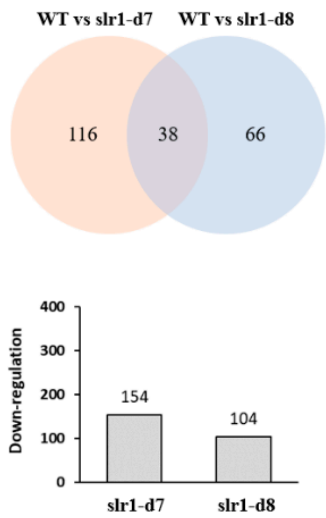

Figure 3. Global gene expression changes in knockout OsSLR1 in rice. (A) Heat map of gene expression between WT vs. slr1-d7 and WT vs. slr1-d8 lines. Red denotes samples with relatively high expression of a given gene and blue denotes samples with relatively low expression. (B) Comparison of the number of differentially expressed genes (DEGs) in WT vs. slr1-d7 and WT vs. slr1-d8. (C) The number of DEGs up- and down-regulated between WT vs. mutant lines.

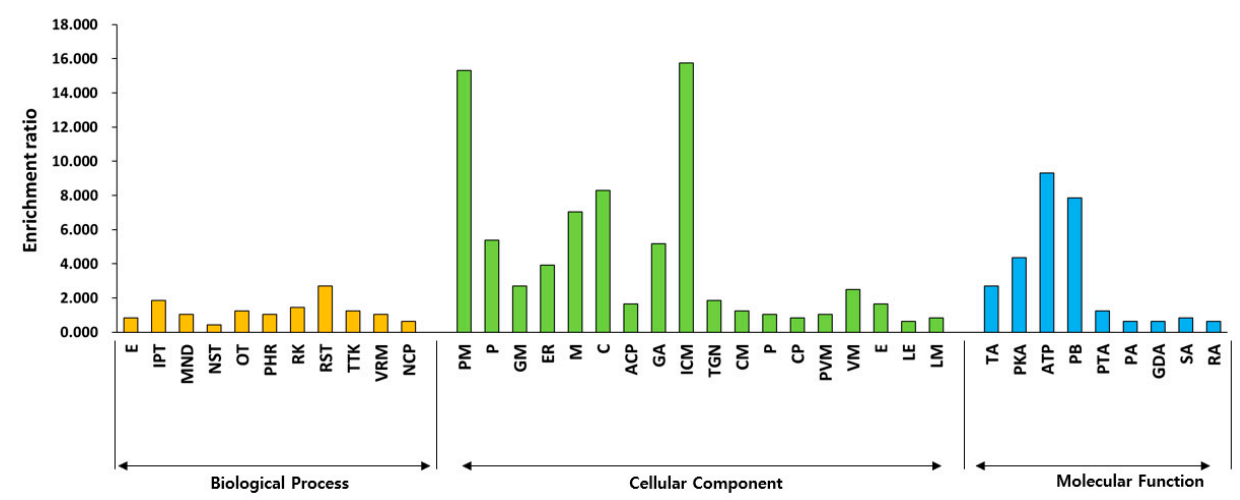

Figure 4. Gene ontology (GO) classification of DEGs shared by WT vs. slr1-d7 and WT vs. slr1-d8 lines. The $x$-axis shows user-selected GO terms, and the $y$-axis shows the enrichment ratio. Biological process: E, exocytosis; IPT, intracellular protein transport; MND, mitotic nuclear division; NST, nitric oxide mediated signal transduction; OT, oligopeptide transport; PHR, plant-type hypersensitive response; RK, response to karrikin; RST, response to salt stress; TTK, transmembrane receptor protein tyrosine kinase signaling pathway; VRM, vegetative to reproductive phase transition of meristem; $\mathrm{NCP}$, nuclear-transcribed mRNA catabolic process. Cellular component: PM, plasma membrane; P, plasmodesma; GM, Golgi membrane; ER, endoplasmic reticulum; M, membrane; C, cytosol; $\mathrm{ACP}$, anchored component of plasma membrane; GA, Golgi apparatus; ICM, integral component of membrane; TGN, trans-Golgi network; $\mathrm{CM}$, chloroplast membrane; $\mathrm{P}$, phragmoplast; $\mathrm{CP}$, cytoplasmic mRNA processing body; PVM, plant-type vacuole membrane; VM, vacuolar membrane; E, endosome; LE, late endosome; EM, endosome membrane. Molecular function: TA, transporter activity; PKA, protein serine/threonine kinase activity; ATP, ATP binding; PB, protein binding; PTA, protein transporter activity; PA, potassium: proton antiporter activity; GDA, glucan endo-1,3-beta-D-glucosidase activity; SA, symporter activity; RA, ribonuclease activity. 
A

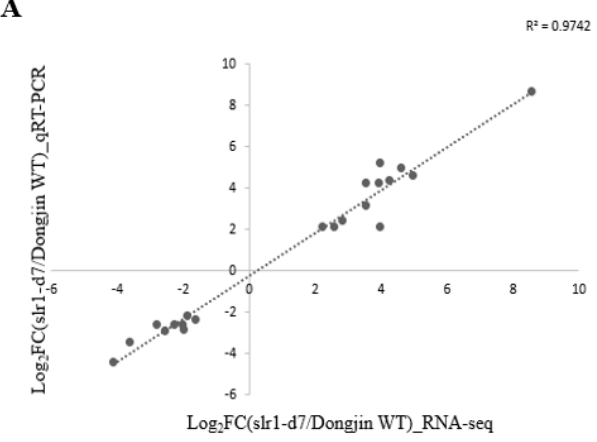

B

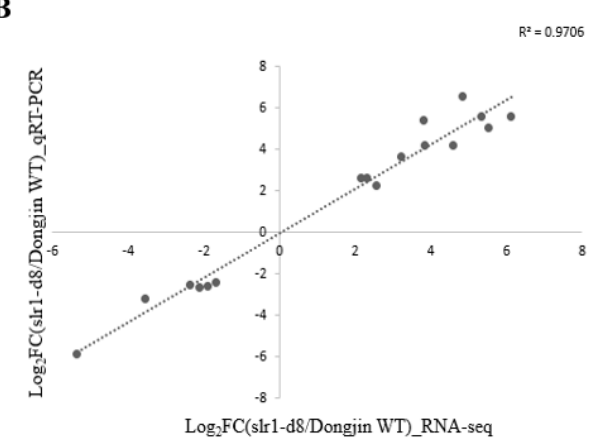

Figure 5. Correlation analysis of gene expression pattern by RNA-Seq and qRT-PCR. (A) WT vs. slr1-d7 line, (B) WT vs. slr1-d8 line.

\subsection{Key DEGs Related to Biosynthesis and Signaling Pathway of Plant Hormone}

In the RNA-seq analysis, the key DEGs related to plant hormone biosynthesis and signal transduction pathways between slr1-d7 vs. WT and slr1-d8 vs. WT were investigated. DEGs between slr1-d7 vs. WT were down-regulated and included the following: gibberellin-regulated protein 2, gibberellin 2-beta-dioxygenase $8\left(G A_{2} O X_{8}\right), E 3$ ubiquitin-protein ligase (XERICO), gibberellin 2-beta-dioxygenase $1\left(G_{2} O X_{1}\right)$ in GA biosynthesis, ERF03, ERF110, BBM1 in ethylene biosynthesis, and ILR1 in auxin biosynthesis. Additionally, DEGs between slr1-d7 vs. WT were up-regulated and included the following: gibberellin 2-beta dioxygenase 8, PIF1, PIF4, $\mathrm{GA}_{20} O \mathrm{OX}_{2}, G A M Y B, G A_{3} O \mathrm{X}_{2}$ in GA biosynthesis, ERF109, ERF39 in ethylene biosynthesis, LOGL1 in cytokinin biosynthesis, IAA7 in the auxin biosynthesis pathway, and JAR1 in jasmonic acid (JA) biosynthesis (Supplementary Table S5). Among the DEGs associated with cell plate and leaf morphogenesis, Os05g0432200 and Os04g0407800 seemed to be important for the difference between WT and edited lines. The expression levels of two GA-related genes, especially $\mathrm{GA}_{20} O \mathrm{OX}_{2}$ (gibberellin oxidase) and $\mathrm{GA}_{3} O \mathrm{OX}_{2}$, were increased in the edited mutant plants compare to WT (Figure 5). The edited mutant lines are required by altering GA responses, at least partially by a defect in the phytohormone signaling process and prevented cell elongation.

\section{Discussion}

CRISPR technology, as a powerful and highly efficient genome editing tool for breeding programs, has been utilized to enable the modification of gene(s) of interest. We report here the CRISPR/Cas9 mutation that potentially could confer a desirable dwarf trait using CRISPR technology. For a long time, plant breeders have used the DELLA gene mutant to reduce plant height [32]. The DELLA protein is one of the main components of the GA signaling pathway and acts as an inhibitor of the GA response. To date, information on dwarfism has shown results in a dominant, semi-dwarf phenotype, such as the observation that the GA signaling repressor DELLA protein or deletion in the N-terminal region suppresses GA signaling [33]. In rice, a total of six slr1-d mutants are known, and these mutants have dark green leaves, reduced internode elongation, and reduced response to GA treatment [28-30,32]. Most slr1-d alleles had $1 \mathrm{bp}$ substitutions, resulting in amino acid substitutions in the conserved TVHYNP motif of SLR1 (Supplementary Figure S6A). In this study, we generated and characterized six new alleles, namely, slr1-d7 slr1-d12, of the dwarf mutants in rice (Supplementary Figure S6B). These mutants showed the same phenotype for leaf color, GA response, and internode elongation with the previously reported slr1-d1 slr1-d6 mutants [28]. Among these mutants, the slr1-d7 gene had a deletion of three nucleotides, resulting in a serine deletion following core sequencing of the TVHYNP motif. Furthermore, the slr1-d8 mutant showed a 1 bp substitution $(+\mathrm{T} /+\mathrm{T})$. These two mutants displayed the most obvious and significant mutant phenotypes. The knockout of the slr1-d8 mutant showed a difference in plant height compared to the deletion of the serine residue of the slr1-d7 mutant (Figure 1, Supplementary Figure S7). This suggests the importance of the TVHYNP domain 
sequence, as the absence of these amino acids affects the normal metabolism of the SLR1 protein, but the degree of reduction is weaker than previously reported for the slr1-d6 mutant series [28]. We also performed paraffin sections to investigate cell length and cell layer using slr1-d7, slr1-d8 and WT. As a result, it was found that slr1-d7 and slr1-d8 not only showed significantly reduced cell length, but also node thickening, as cell layers increased as compared to WT (Figure 2). In addition, these dwarf mutants showed a decrease in the whole internode length containing a panicle when compared to WT. This result is similar to the characteristics of $d n$-type rice dwarf mutants reported by Takeda [31] and, as a semi-dominant, a decrease in cell length may be a direct cause of shortening clum length in dwarf mutant plants. In shoot elongation tests of dwarf mutant reaction by exogenous $\mathrm{GA}_{3}$, the length of the secondary leaf sheath was elongated by GA treatment in WT, but not observed in slr1-d7 and slr1-d8 lines. However, there was a difference between the mutations. These results were similar to those reported in barley $\operatorname{Sin} 1 D$ and corn $\operatorname{dwarf} 8$, suggesting that a single amino acid deletion or exchange mutation showed an intermediate phenotype depending on plant growth and GAI protein stabilization $[13,17,34]$. In RNA-seq analysis, the key DEGs related to plant hormone biosynthesis and the signal transduction pathways between slr1-d7 vs. WT and slr1-d8 vs. WT were investigated. DEGs between slr1-d7 vs. WT were down-regulated and included the following: gibberellin-regulated protein 2, gibberellin 2-beta-dioxygenase $8\left(\mathrm{GA}_{2} \mathrm{OX}_{8}\right)$, E3 ubiquitin-protein ligase (XERICO), gibberellin 2-beta-dioxygenase $1\left(G A_{2} O X_{1}\right)$ in GA biosynthesis [35], ERF03, ERF110, BBM1 in ethylene biosynthesis [36], and ILR1 in auxin biosynthesis [37]. In RT-PCR and RNA-seq analysis, the expression levels of two GA-related genes, $\mathrm{GA}_{20} O \mathrm{O}_{2}$ and $\mathrm{GA}_{3} O \mathrm{OX}_{2}$, increased in the edited mutant line compared to $\mathrm{WT}$, suggesting that these genes convert in the $\mathrm{GA}_{12}$ signaling system (Figure 5). The phenomenon of inhibiting cell elongation by altering the GA response due to defects in the signal transduction process of plant hormones was consistent with the results of the Arabidopsis mutants [35]. Furthermore, DEGs between slr1-d7 vs. WT were up-regulated and included the following: gibberellin 2-beta dioxygenase 8, PIF1, PIF4, GA ${ }_{20} \mathrm{OX}_{2}, \mathrm{GAMYB}, \mathrm{GA}_{3} \mathrm{OX}_{2}$ in GA biosynthesis [38-43], ERF109, ERF39 in ethylene biosynthesis [36], LOGL1 in cytokinin biosynthesis [44], IAA7 in the auxin biosynthesis pathway [45], and JAR1 in jasmonic acid (JA) biosynthesis [46]. In summary, our results showed that slr1-d7 and slr1-d8 caused a defect in the phytohormone signaling system process and prevented cell elongation. Furthermore, we suggested that the new slr1-d7 slr1-d12 allelic variants are valuable semi-dominant dwarf alleles with potential application value for molecule breeding using the CRISPR/Cas9 system in rice.

\section{Materials and Methods}

\subsection{Plant Materials}

Rice variety Dongjin (Oryza sativar L., ssp. Japonica) was used for transformation experiments. Plants were grown in GMO greenhouse facilities and rice fields at Hankyong National University in Korea. Harvested seeds were dried to $\sim 14 \%$ moisture content and kept in dry conditions at $4{ }^{\circ} \mathrm{C}$.

\subsection{CRISPR/Cas9 Vector Construction and Rice Transformation}

SgRNAs were designed as described in Park et al. [47] to target the TVHYNP motif. The TVHYNPSD amino acid sequence of the SLR1 gene is encoded by ACCGTGCACTACAACCCCTCGGAC, and the target sequence ACCCCTCGGACCTCTCCCTCCTGG with TGG as the PAM was selected. The 20nt sgRNA scaffold sequence was synthesized by Bioneer co., LTD (Dajeon, Korea). The slr-sgRNA templates were annealed using two primers, $5^{\prime}$-ggcagACCCCTCGGACCTCTCCTCC-3' and 5'-aaacGGAGGAGAGGTCCGAGGGGTc-3', and cloned into an AarI-digested OsU3:pBOsC binary vector. The Ti-plasmid vector for sgRNA expression, OsU3:slr1-sgRNA/pBOsC, and its flanking sequences were confirmed by the Sanger sequencing method and mobilized into Agrobacterium-tumefaciens strain EHA105. Transgenic plants were regenerated following a previously described protocol [48]. To confirm the transgene, the independent and transformed lines were 
analyzed by PCR. Plants derived from tissue culture were rooted and potted into $7 \mathrm{~cm}$ pots placed in the glasshouse and gradually acclimatized to the glasshouse conditions.

\subsection{Targeted Deep Sequencing and Mutation Analysis}

Total DNA extraction from plant tissues was performed using the DNA Quick Plant Kit (Inclone, Korea). Targeted deep sequencing analysis was performed following the method described by Jung et al. [46]. All primers used for targeted deep sequencing are listed in Supplementary Table S1. Paired-end read sequencing by PCR amplicons was produced with MiniSeq (Illumina, San Diego, CA, USA). All data derived from MiniSeq were analyzed by Cas-Analyzer (http://www.rgenome.net/casanalyzer), as previously reported by Park et al. [49].

\subsection{RNA-Seq and Data Analysis}

To investigate the transcriptome of edited lines obtained by the OsSLR1 gene via the CRISPR/Cas9 system, WT, slr1-d7(T/T), and slr1-d8 (-3/-3) plants were used for RNA-seq analysis. Four-week-old leaf tissues were used for RNA extraction, as previously reported by Wang et al. [50]. RNA concentration (A260/A280 and A260/A230) was measured with spectrophotometry (Nanodrop 2000, Thermo Scientific, Hudson, NH, USA). A Bioanalyzer (Agilent Technologies, Santa Clara, CA, USA) was used to evaluate the RNA qualities. Leaf samples of $100 \mathrm{mg}$ were collected from three plants (WT, slr1-d7, and slr1-d8) for RNA-seq analysis. RNA-sequencing was carried out by Macrogen (Seoul, Korea, https: //dna.macrogen.com/). Clean reads were produced by removing low-quality reads and mapped to the reference genome (https://plants.ensembl.org/) using TopHat2 (https://ccb.jhu.edu/software/tophat) [51]. Based on location information of the mapped reads, gene expression levels were normalized to reads per kilobase per million mapped reads (RPKM). DEG analyses between the edited plant RNA (slr1-d7, slr1-d8 and WT) were performed using the standard fold change (FC) $\geq 2$ and FDR $<0.05$. GO analysis was performed as previously reported by Chow et al. [52].

\subsection{Validation Test of Selected DEGs}

To validate the accuracy of the RNA-sequencing data, qRT-PCR was conducted on twenty-one selected genes. The slr1-d7 and slr1-d8 lines were assessed according to $\mathrm{WT}$, and relative gene expression levels were normalized by the Actin gene (XM_015761709). All assays for each gene were performed in triplicate with the same conditions and the RNA-seq data were deposited into the NCBI database.

\section{6. $G A_{3}$ Treatment}

The slr1-d7, slr1-d8, and WT seedlings grown in pots for 4 weeks were sprayed with $50 \mu \mathrm{M} \mathrm{GA}_{3}$ (Sigma-Aldrich, Seoul, Korea). The stock solution of $\mathrm{GA}_{3}$ was dissolved in ethanol and added to autoclaved water after cooling to approximately $60^{\circ} \mathrm{C}$ to make the final $50 \mu \mathrm{M}$ solution. The WT plants were treated with water containing equal amounts of ethanol.

\subsection{Light Microscopy}

For the paraffin section, stems and leaves were harvested from slr1-d7, slr1-d8, and WT plants. First, stem tissues were treated with $15 \%$ hydrofluoric acid, followed by dehydration with $70 \%$ ethanol, removal of it, infiltration, and embedding. For imaging, a $10 \mu \mathrm{m}$ microtome section was placed on glass slides and floated in a $37^{\circ} \mathrm{C}$ water bath containing deionized water. The sections were floated onto clean glass slides and microwaved at $65^{\circ} \mathrm{C}$ for $15 \mathrm{~min}$. Following this, the tissues were bound to the glass and each slide was used in chemical staining immediately.

Supplementary Materials: Supplementary Materials can be found at http:/www.mdpi.com/1422-0067/21/15/ 5492/s1. Figure S1. Nucleotide and amino acid sequences of the SLR1 gene in rice. Figure S2. Amino acid sequence alignment of the coding region of the SLR1 gene from Oryza sativa, Sorghum bicolor, Zea mays, Panicum miliaceum and Triticum aestivum. Figure S3. Confirmation of the efficiency of sgRNA using T7-endonuclease I 
enzyme. Figure S4. CRISPR/Cas9 binary vector construction and rice transformation. Figure S5. Confirmation of the mutant phenotype and the conserved domain region of the DELLA protein. Figure S6. CRISPR/Cas9-induced mutations in the OsSLR1 gene and the phenotype of the edited plants. Figure S7. Appearance of the panicle, leaves and grains after harvesting slr1-d7 and slr1-d8 lines compared to WT. Table S1. Design of sgRNAs for CRISPR genome editing on the OsSRL1 gene in rice. Table S2. Mutation rate and edited plant types for the OsSLR1 gene using the CRISPR/Cas9 system. Table S3. GO enrichment analysis of DEGs by RNA-seq analysis. Table S4. Key DEGs of up- and down-regulated genes related to phytohormone biosynthesis and signaling transduction pathway by RNA seqs in WT vs slr1-d7 and WT vs slr1-d8 lines. Table S5. List of primers and gene sequences in DEGs, correlation analysis of gene expression pattern by RNA-Seq and qRT-PCR.

Author Contributions: Formal analysis, Y.J.J., J.H.K., H.J.L., D.H.K. and J.Y.; investigation, Y.-G.C.; resources, S.B. and K.K.K.; software, H.J.L., J.Y. and S.B.; supervision, K.K.K.; writing-original draft, Y.J.J.; writing-review and editing, K.K.K. All authors have read and agreed to the published version of the manuscript.

Funding: This research was funded by "Cooperative Research Program for Agriculture Science \& Technology Development (Project No. PJ01477203)" Rural Development Administration, Korea.

Conflicts of Interest: The authors declare no conflict of interest. The funders had no role in the design of the study; in the collection, analyses, or interpretation of data; in the writing of the manuscript, or in the decision to publish the results.

\section{References}

1. Wang, Y.; Li, J. The plant architecture of rice (Oryza sativa). Plant Mol. Biol. 2005, 59, 75-84. [CrossRef]

2. Yamaguchi, S. Gibberellin metabolism and its regulation. Annu. Rev. Plant Biol. 2008, 59, 225-251. [CrossRef]

3. Sun, T.P. The molecular mechanism and evolution of the GA-GID1-DELLA signaling module in plants. Curr. Biol. 2011, 21, R338-R345. [CrossRef] [PubMed]

4. Sakamoto, T.; Miura, K.; Itoh, H.; Tatsumi, T.; Ueguchi-Tanaka, M.; Ishiyama, K.; Miyao, A. An overview of gibberellin metabolism enzyme genes and their related mutants in rice. Plant Physiol. 2004, 134, 1642-1653. [CrossRef] [PubMed]

5. Itoh, H.; Ueguchi-Tanaka, M.; Sentoku, N.; Kitano, H.; Matsuoka, M.; Kobayashi, M. Cloning and functional analysis of two gibberellin $3 \beta$-hydroxylase genes that are differently expressed during the growth of rice. Proc. Natl. Acad. Sci. USA 2001, 98, 8909-8914. [CrossRef]

6. Itoh, H.; Tatsumi, T.; Sakamoto, T.; Otomo, K.; Toyomasu, T.; Kitano, H.; Matsuoka, M. A rice semi-dwarf gene, Tan-Ginbozu (D35), encodes the gibberellin biosynthesis enzyme, ent-kaurene oxidase. Plant Mol. Biol. 2004, 54, 533-547. [CrossRef]

7. Monna, L.; Kitazawa, N.; Yoshino, R.; Suzuki, J.; Masuda, H.; Maehara, Y.; Minobe, Y. Positional cloning of rice semidwarfing gene, $s d-1$ : Rice "green revolution gene" encodes a mutant enzyme involved in gibberellin synthesis. DNA Res. 2002, 9, 11-17. [CrossRef]

8. Zhu, Y.; Nomura, T.; Xu, Y.; Zhang, Y.; Peng, Y.; Mao, B.; Zhu, X. ELONGATED UPPERMOST INTERNODE encodes a cytochrome $\mathrm{P} 450$ monooxygenase that epoxidizes gibberellins in a novel deactivation reaction in rice. Plant Cell 2006, 18, 442-456. [CrossRef]

9. Hirano, K.; Nakajima, M.; Asano, K.; Nishiyama, T.; Sakakibara, H.; Kojima, M.; Banks, J.A. The GID1-mediated gibberellin perception mechanism is conserved in the lycophyte Selaginella moellendorffii but not in the bryophyte Physcomitrella patens. Plant Cell 2007, 19, 3058-3079. [CrossRef]

10. Yasumura, Y.; Crumpton-Taylor, M.; Fuentes, S.; Harberd, N.P. Step-by-step acquisition of the gibberellin-DELLA growth-regulatory mechanism during land-plant evolution. Curr. Biol. 2007, 17, 1225-1230. [CrossRef]

11. Peng, J.; Carol, P.; Richards, D.E.; King, K.E.; Cowling, R.J.; Murphy, G.P.; Harberd, N.P. The Arabidopsis GAI gene defines a signaling pathway that negatively regulates gibberellin responses. Genes Dev. 1997, 11, 3194-3205. [CrossRef] [PubMed]

12. Ikeda, A.; Ueguchi-Tanaka, M.; Sonoda, Y.; Kitano, H.; Koshioka, M.; Futsuhara, Y.; Yamaguchi, J. slender rice, a constitutive gibberellin response mutant, is caused by a null mutation of the SLR1 gene, an ortholog of the height-regulating gene GAI/RGA/RHT/D8. Plant Cell 2001, 13, 999-1010. [CrossRef] [PubMed]

13. Chandler, P.M.; Marion-Poll, A.; Ellis, M.; Gubler, F. Mutants at the Slender1 locus of barley cv Himalaya. Molecular and physiological characterization. Plant Physiol. 2002, 129, 181-190. [CrossRef]

14. Hirano, K.; Ueguchi-Tanaka, M.; Matsuoka, M. GID1-mediated gibberellin signaling in plants. Trends Plant Sci. 2008, 13, 192-199. [CrossRef] 
15. Ueguchi-Tanaka, M.; Ashikari, M.; Nakajima, M.; Itoh, H.; Katoh, E.; Kobayashi, M.; Matsuoka, M. GIBBERELLIN INSENSITIVE DWARF1 encodes a soluble receptor for gibberellin. Nature 2005, 437, 693-698. [CrossRef]

16. Sun, T.P. Gibberellin-GID1-DELLA: A pivotal regulatory module for plant growth and development. Plant Physiol. 2010, 154, 567-570. [CrossRef]

17. Willige, B.C.; Ghosh, S.; Nill, C.; Zourelidou, M.; Dohmann, E.M.N.; Maier, A.; Schwechheimer, C. The DELLA domain of GA INSENSITIVE mediates the interaction with the GA INSENSITIVE DEARF1A gibberellin receptor of Arabidopsis. Plant Cell 2007, 19, 1209-1220. [CrossRef]

18. Epstein, E.; Bloom, A. Mineral Nutrition of Plants: Principles and Perspectives, 2nd ed.; Sinauer Associates: Sunderland, MA, USA, 2005; p. 400.

19. Liang, Y.; Sun, W.; Zhu, Y.G.; Christie, P. Mechanisms of silicon-mediated alleviation of abiotic stresses in higher plants: A review. Environ. Pollut. 2007, 147, 422-428. [CrossRef]

20. Harberd, N.P.; Belfield, E.; Yasumura, Y. The angiosperm gibberellin-GID1-DELLA growth regulatory mechanism: How an "inhibitor of an inhibitor" enables flexible response to fluctuating environments. Plant Cell 2009, 21, 1328-1339. [CrossRef]

21. Guntzer, F.; Keller, C.; Meunier, J.D. Benefits of plant silicon for crops: A review. Agron. Sustain. Dev. 2012, 32, 201-213. [CrossRef]

22. Rodrigues, F.A.; Datnoff, L.E. Silicon and rice disease management. Fitopatol. Bras. 2005, 30, 457-469. [CrossRef]

23. Alagoz, Y.; Gurkok, T.; Zhang, B.; Unver, T. Manipulating the biosynthesis of bioactive compound alkaloids for next-generation metabolic engineering in opium poppy using CRISPR-Cas 9 genome editing technology. Sci. Rep. 2016, 6, 30910. [CrossRef]

24. Liu, X.; Wu, S.; Xu, J.; Sui, C.; Wei, J. Application of CRISPR/Cas9 in plant biology. Acta Phys. Sin. 2017, 7, 292-302. [CrossRef]

25. Hayut, S.F.; Bessudo, C.M.; Levy, A.A. Targeted recombination between homologous chromosomes for precise breeding in tomato. Nat. Commun. 2017, 8, 1-9.

26. Čermák, T.; Curtin, S.J.; Gil-Humanes, J.; Čegan, R.; Kono, T.J.; Konečná, E.; Voytas, D.F. A multipurpose toolkit to enable advanced genome engineering in plants. Plant Cell 2017, 29, 1196-1217. [CrossRef]

27. Bae, S.; Park, J.; Kim, J.S. Cas-OFFinder: A fast and versatile algorithm that searches for potential off-target sites of Cas9 RNA-guided endonucleases. Bioinformatics 2014, 30, 1473-1475. [CrossRef]

28. Asano, K.; Hirano, K.; Ueguchi-Tanaka, M.; Angeles-Shim, R.B.; Komura, T.; Satoh, H.; Ashikari, M. Isolation and characterization of dominant dwarf mutants, Slr1-d, in rice. Mol. Genet. Genom. 2009, 281, 223-231. [CrossRef]

29. Hirano, K.; Asano, K.; Tsuji, H.; Kawamura, M.; Mori, H.; Kitano, H.; Matsuoka, M. Characterization of the molecular mechanism underlying gibberellin perception complex formation in rice. Plant Cell 2010, 22, 2680-2696. [CrossRef]

30. Zhang, Y.H.; Bian, X.F.; Zhang, S.B.; Ling, J.; Wang, Y.J.; Wei, X.Y.; Fang, X.W. Identification of a novel gain-of-function mutant allele, slr1-d5, of rice DELLA protein. J. Integr. Agric. 2016, 15, 1441-1448. [CrossRef]

31. Takeda, K. Internode elongation and dwarfism in some graminaeous plants. Gamma Field Symp. 1977, 16, 1-18.

32. Wu, Z.; Tang, D.; Liu, K.; Miao, C.; Zhuo, X.; Li, Y.; Cheng, Z. Characterization of a new semi-dominant dwarf allele of SLR1 and its potential application in hybrid rice breeding. J. Exp. Bot. 2018, 69, 4703-4713. [CrossRef] [PubMed]

33. Peng, J.; Richards, D.E.; Hartley, N.M.; Murphy, G.P.; Devos, K.M.; Flintham, J.E.; Sudhakar, D. ‘Green revolution'genes encode mutant gibberellin response modulators. Nature 1999, 400, 256-261. [CrossRef] [PubMed]

34. Gubler, F.; Chandler, P.M.; White, R.G.; Llewellyn, D.J.; Jacobsen, J.V. Gibberellin signaling in barley aleurone cells. Control of SLN1 and GAMYB expression. Plant Physiol. 2002, 129, 191-200. [CrossRef]

35. Ko, J.H.; Yang, S.H.; Han, K.H. Upregulation of an Arabidopsis RING-H2 gene, XERICO, confers drought tolerance through increased abscisic acid biosynthesis. Plant J. 2006, 47, 343-355. [CrossRef]

36. Boutilier, K.; Offringa, R.; Sharma, V.K.; Kieft, H.; Ouellet, T.; Zhang, L.; Custers, J.B. Ectopic expression of BABY BOOM triggers a conversion from vegetative to embryonic growth. Plant Cell 2002, 14, 1737-1749. [CrossRef] 
37. Widemann, E.; Miesch, L.; Lugan, R.; Holder, E.; Heinrich, C.; Aubert, Y.; Heitz, T. The amidohydrolases IAR3 and ILL6 contribute to jasmonoyl-isoleucine hormone turnover and generate 12-hydroxyjasmonic acid upon wounding in Arabidopsis leaves. J. Biol. Chem. 2013, 288, 31701-31714. [CrossRef]

38. Schomburg, F.M.; Bizzell, C.M.; Lee, D.J.; Zeevaart, J.A.; Amasino, R.M. Overexpression of a novel class of gibberellin 2-oxidases decreases gibberellin levels and creates dwarf plants. Plant Cell 2003, 15, 151-163. [CrossRef]

39. Huq, E.; Al-Sady, B.; Hudson, M.; Kim, C.; Apel, K.; Quail, P.H. Phytochrome-interacting factor 1 is a critical bHLH regulator of chlorophyll biosynthesis. Science 2004, 305, 1937-1941. [CrossRef]

40. Pedmale, U.V.; Huang, S.S.C.; Zander, M.; Cole, B.J.; Hetzel, J.; Ljung, K.; Ecker, J.R. Cryptochromes interact directly with PIFs to control plant growth in limiting blue light. Cell 2016, 164, 233-245. [CrossRef] [PubMed]

41. Rieu, I.; Ruiz-Rivero, O.; Fernandez-Garcia, N.; Griffiths, J.; Powers, S.J.; Gong, F.; Phillips, A.L. The gibberellin biosynthetic genes $A t G A_{20} O x_{1}$ and $A t G A_{20} O x_{2}$ act, partially redundantly, to promote growth and development throughout the Arabidopsis life cycle. Plant J. 2008, 53, 488-504. [CrossRef] [PubMed]

42. Kaneko, M.; Inukai, Y.; Ueguchi-Tanaka, M.; Itoh, H.; Izawa, T.; Kobayashi, Y.; Matsuoka, M. Loss-of-function mutations of the rice $G A M Y B$ gene impair $\alpha$-amylase expression in aleurone and flower development. Plant Cell 2004, 16, 33-44. [CrossRef] [PubMed]

43. Yamaguchi, S.; Smith, M.W.; Brown, R.G.; Kamiya, Y.; Sun, T.P. Phytochrome regulation and differential expression of gibberellin 3 $\beta$-hydroxylase genes in germinating Arabidopsis seeds. Plant Cell 1998, 10, 2115-2126. [CrossRef] [PubMed]

44. Kuroha, T.; Tokunaga, H.; Kojima, M.; Ueda, N.; Ishida, T.; Nagawa, S.; Sakakibara, H. Functional analyses of LONELY GUY cytokinin-activating enzymes reveal the importance of the direct activation pathway in Arabidopsis. Plant Cell 2009, 21, 3152-3169. [CrossRef]

45. Liscum, E.; Reed, J.W. Genetics of Aux/IAA and ARF action in plant growth and development. Plant Mol. Biol. 2002, 49, 387-400. [CrossRef]

46. Pena-Cortés, H.; Albrecht, T.; Prat, S.; Weiler, E.W.; Willmitzer, L. Aspirin prevents wound-induced gene expression in tomato leaves by blocking jasmonic acid biosynthesis. Planta 1993, 191, 123-128. [CrossRef]

47. Park, J.; Bae, S.; Kim, J.S. Cas-Designer: A web-based tool for choice of CRISPR-Cas9 target sites. Bioinformatics 2015, 31, 4014-4016. [CrossRef]

48. Jung, Y.J.; Lee, H.J.; Kim, J.H.; Kim, D.H.; Kim, H.K.; Cho, Y.G.; Kang, K.K. CRISPR/Cas9-targeted mutagenesis of $F 3^{\prime} H, D F R$ and LDOX, genes related to anthocyanin biosynthesis in black rice (Oryza sativa L.). Plant Biotechnol. Rep. 2019, 13, 521-531. [CrossRef]

49. Park, J.; Lim, K.; Kim, J.S.; Bae, S. Cas-analyzer: An online tool for assessing genome editing results using NGS data. Bioinformatics 2017, 33, 286-288. [CrossRef]

50. Wang, L.; Feng, Z.; Wang, X.; Wang, X.; Zhang, X. DEGseq: An R package for identifying differentially expressed genes from RNA-seq data. Bioinformatics 2010, 26, 136-138. [CrossRef]

51. Kim, K.H.; Kang, Y.J.; Shim, S.; Seo, M.J.; Baek, S.B.; Lee, J.H.; Park, S.K.; Jun, T.H.; Moon, J.K.; Lee, S.H.; et al. Genome-wide RNA-seq analysis of differentially expressed transcription factor genes against bacterial leaf pustule in soybean. Plant Breed. Biotech. 2015, 3, 197-207. [CrossRef]

52. Chow, K.S.; Wan, K.L.; Isa, M.N.M.; Bahari, A.; Tan, S.H.; Harikrishna, K.; Yeang, H.Y. Insights into rubber biosynthesis from transcriptome analysis of Hevea brasiliensis latex. J. Exp. Bot. 2007, 58, 2429-2440. [CrossRef] [PubMed]

(C) 2020 by the authors. Licensee MDPI, Basel, Switzerland. This article is an open access article distributed under the terms and conditions of the Creative Commons Attribution (CC BY) license (http://creativecommons.org/licenses/by/4.0/). 